\title{
Carnets
}

Revue électronique d'études françaises de l'APEF

Deuxième série - $18 \mid 2020$

Chiens et écritures (littéraires, filmiques, photographiques)

\section{La représentation du chien dans le roman policier francophone : compagnon, enquêteur, victime ou bourreau?}

Pierre Michel Pranville

\section{OpenEdition}

\section{Journals}

Édition électronique

URL : http://journals.openedition.org/carnets/10825

DOI : 10.4000/carnets. 10825

ISSN : 1646-7698

Éditeur

APEF

Référence électronique

Pierre Michel Pranville, «La représentation du chien dans le roman policier francophone : compagnon, enquêteur, victime ou bourreau? », Carnets [En ligne], Deuxième série - 18 | 2020, mis en ligne le 31 janvier 2020, consulté le 03 juin 2020. URL : http://journals.openedition.org/carnets/10825 ; DOI : https://doi.org/10.4000/carnets.10825

Ce document a été généré automatiquement le 3 juin 2020

\section{(c) (†) \&)}

Carnets est mis à disposition selon les termes de la licence Creative Commons - Atribution - Pas d'utilisation commerciale 4.0 International. 


\title{
La représentation du chien dans le roman policier francophone : compagnon, enquêteur, victime ou bourreau?
}

\author{
Pierre Michel Pranville
}

1 L'image du chien policier qui flaire une piste, à la recherche de la victime ou de l'assassin, familière des séries télévisées, n'est plus représentée dans le roman policier contemporain où l'homme semble renoncer à une partie de sa suprématie sur l'animal qui acquiert d'autres dimensions beaucoup plus valorisantes: celle d'un acteur de l'intrigue, positif ou maléfique, ou parfois celle d'un personnage à part entière au rôle plus ou moins important. Le premier contact avec le roman policier est son titre et sa couverture, et le chien y est très présent même s'il peut disparaitre du contenu une fois la couverture tournée. Dans ce cas, le chien en titre, le chien-titre, informe sur le climat du roman et annonce des expériences ou des comportements humains tels que l'infortune, la lâcheté, la violence, l'horreur (Le chien de minuit, Chiennes, Prendre les loups pour des chiens) ou, au contraire, la fidélité, la résistance, la détermination, le courage (La part des chiens, Lâchez les chiens, Un temps de chien), et fait directement appel à l'imaginaire lié à l'animal par le lecteur.

2 Si le chien confirme sa présence dans l'intrigue une fois la couverture tournée, alors il convient d'étudier quelle est sa fonction : est-il un animal de compagnie, l'auxiliaire d'un enquêteur, le témoin de l'histoire racontée ou un personnage autonome qu'il soit héros, victime ou bourreau. Nous observerons dans un premier temps si cette typologie des fonctions correspond à une typologie des figures du chien habituellement identifiées dans la mythologie et dans la littérature : le chien protecteur, loyal, le chien démoniaque, perturbateur. Nous conclurons en étudiant s'il y a un lien ou non entre le chien acteur et figure positive ou maléfique et les différents sous-genres du roman policier : le roman à énigme, le roman noir, le roman d'angoisse, sans oublier le polar parodique. Pour cela, nous nous appuierons sur un corpus francophone (Belgique, 
France, Suisse) de sept romans du Chien jaune de Simenon (1936), au Chien arabe de Benoit Severac (2017). Nous vérifierons que, dans le roman policier, en général, les rapports entre l'homme et le chien développent une sophistication inattendue porteuse d'une coopération entre espèces humaine et animale.

Trois figures du chien prédominent dans la mythologie et dans la littérature : le gardien, le protecteur, un compagnon obéissant, intègre et fidèle. Le meilleur ami de l'homme avec lequel il communique. Il est un trait d'union entre le monde animal et l'homme; le défenseur mais aussi l'attaquant : il est celui qui piste les ennemis. Il met à la disposition de l'homme des sens plus développés que les siens comme l'odorat; le chien monstrueux, maléfique, féroce : associé à la mort qu'il annonce ou qu'il donne. Il renvoie à l'image de Cerbère, le chien à trois têtes, le gardien des Enfers, ou à celle attachée à l'Egypte ancienne, de passeur vers le royaume des morts. Nous retrouvons ces trois figures dans la littérature policière et nous observerons qu'elles coïncident avec les trois fonctions du chien relevées dans les romans policiers de notre corpus. Elles sont définies par le degré d'autonomie du chien vis à vis de l'homme : d'abord le chien compagnon de son maître est présent, fidèle, loyal. Il a le rôle d'un confident, parfois d'un conseiller. Puis le chien compagnon et co-enquêteur de son maitre est indispensable à la bonne marche de l'enquête. Il dialogue avec le policier ou l'agent secret qu'il épaule efficacement. Sans ce chien aux comportements humains, il n'y a pas de succès dans l'enquête ou dans la mission; enfin, sans dimension anthropomorphique, les chiens porteurs d'un message, et qui sont rarement des héros, plus souvent des victimes d'hommes qui les ont dressés à être violents, ou bien ils annoncent le chaos et la mort.

Dans La nuit myope d'A.D.G. pseudonyme d'Alain Fournier, publié en 1990, le chien est un compagnon attentionné de son maitre, mu par des sentiments tout à fait humains. Cette nouvelle raconte une traversée nocturne de Paris par Domi, cadre, la quarantaine, ivre, myope, qui a brisé ses lunettes, accompagné de son chien Laskar, un berger belge. Domi, séduit par Armelle dans une boite de nuit veut quitter femme et travail pour fuir avec elle sur les sentiers de grande randonnée du sud de la France. Le chien Laskar va guider dans la ville noire son bourgeois de maître déboussolé, et remplir tour à tour la fonction de chien d'aveugle, de confident et de conseiller, mauvais conseiller lui enjoignant de tout quitter, ne pensant qu'à son intérêt :

Laskar dardait sur lui des yeux implorants. Te laisse pas faire, maitre, va ta route, les p'tits lapins dans le serpolet n'attendent plus que nous pour battre des pattes arrière contre les troncs des vieux sapins! Suis ton chemin qui est le mien : fumet de marcassin, bruyère écrasée, pets de chevreuil, senteur morne des marrons éclatés... (A.D.G., $1990:$ 76)

5 En fait, Laskar, chien urbain, n'aspire qu'à courir la garrigue et adopte une position totalement amorale et humaine, amorale parce qu'humaine. A.D.G est un auteur français atypique dans le polar des années 1970-1990 et pas seulement parce qu'il revendique sa proximité avec l'extrême-droite dans un genre littéraire très à gauche, même si ses thèmes sont socialement critiques. C'est par son style d'écriture recherché, plein d'humour, de jeux de mots décapants et de néologismes hilarants qu'il se distingue, comme dans ce court roman qui renvoie à la fois au noir social et au roman d'angoisse.

6 Tequila frappée est un roman de Nadine Monfils, auteure belge, qui écrit depuis 1984 . Elle l'a publié en 2009. Tequila est une chienne qui a une vie assez semblable à celle des hommes et porte comme eux des fardeaux : l'assassinat de ses premiers maîtres dont 
elle a été témoin, elle est alcoolique... L'inspecteur Lynch l'a recueillie chez une vieille dame qui venait de mourir.

Les chiennes sont têtues. Surtout ce genre de petite merdouille, croisement d'une saucisse et d'un plumeau avec une gueule de fox-terrier et des yeux de papillon. Ils avaient dû s'y mettre à plusieurs pour arriver à un tel résultat (...). Il avait tout fait pour éviter les câlins, léchouilles et autres effusions intempestives. Peine perdue ! Chaque fois qu'il rentrait du boulot, l'animal bondissait sur lui et le «parfumait » de ses relents à faire rougir un munster oublié sur une plage. Jusqu'au soir où il s'était passé quelque chose d'étonnant entre la chienne et son nouveau maître... (Monfils, $2009: 13-14)$

7 La chienne est montée sur la table basse et a bu la tequila que s'était préparée Lynch. Depuis, l'inspecteur et sa chienne, baptisée illico Tequila, prennent ensemble l'apéritif tous les soirs. Tequila prend à revers l'image du chien racé et bien dressé. Elle adopte des comportements humains qu'elle singe. Elle est la compagne indispensable de son maitre, sa confidente, le fil rouge de la vie de l'homme, accidentée comme la sienne, comme celle de toute la société détraquée qui les entoure. Mais Tequila n'est pas associée à l'enquête rocambolesque que mène l'inspecteur Lynch. Outre la poursuite d'un criminel qui multiplie les assassinats, Lynch est à la recherche de son frère qui s'est enfui de l'hôpital psychiatrique où il est soigné parce qu'il assassine des animaux domestiques. Dans le dernier chapitre, c'est Tequila qui parle et raconte ses expériences successives de maîtres, un récit qu'elle conclut par :

Ici, c'est le top ! (...) On picole tous les soirs. C'est la fête ! J'ai bien mérité ça, parce que, sincèrement j'ai eu une chienne de vie. Pour ça que je suis un peu dingo. Pour supporter les souvenirs. Tout le monde n'a pas la chance de devenir amnésique.

Je porte bien mon nouveau nom : Tequila frappée. (Monfils, 2009 : 283)

8 La chienne Tequila franchit deux étapes vers le genre humain : elle a la mémoire de ses souffrances et elle est devenue narratrice. Notons que celle qui est présente dans le roman ressemble beaucoup à celle qui est représentée sur la couverture qui n'est autre qu'une photo de la chienne de l'auteur, dont elle ne se sépare jamais en interview ou dans un salon littéraire. Comme dans La nuit myope, le chien (la chienne) est ici une compagne sensible, intelligente, un garde-fou face aux incohérences du monde extérieur.

9 De compagnon de son maitre à acteur de l'enquête, le chien de Jean-Hugues Oppel et Xavier Dorison et celui de Frédéric Dard progressent dans l'anthropomorphisme en développant des pensées et des attitudes de plus en plus humaines. Canine et Gunn de Jean-Hugues Oppel et Xavier Dorison a été publié en 1983. C'est un premier roman pour les deux co-auteurs, une parodie de la veine noire d'espionnage de l'époque. Oppel deviendra un auteur de polar (plutôt politique) reconnu, Dorison, lui, s'orientera avec succès vers les scenarii de bande dessinée. Déjà, dès le début, dès le titre, il y a inversion des genres homme-animal. Canine est le nom d'une femme, un agent secret redoutablement mordante, et Gunn, celui de son chien, lui aussi très efficace, autant qu'un gun $\neg$ fusil, avec un seul $\mathrm{n} \neg$. Ce roman initiatique met en scène un chien issu des amours d'un berger belge et d'un lévrier qui se comporte comme un humain: il dialogue avec sa maitresse, il drague effrontément les chiennes à la manière des machos latins, il boit aussi du champagne $\neg$ encore un chien alcoolique? Il est autonome et revendique son indépendance par le refus d'un collier, et il est plein d'initiative dans son travail d'assistant d'un agent de choc, faisant profiter l'homme de sens dont il ne dispose pas, l'ouïe et l'odorat : 
Gunn, un peu à l'écart dans un fourré, poussa un bref gémissement auquel canine répondit par un claquement de langue agacé.

Il n'échappera à personne que ce bref dialogue pouvait se traduire à peu près de la manière suivante :

Merci, mais essaie d'être un peu plus discret quand tu me préviens. (Oppel- Dorison, $1983: 18$ )

10 En mission sur le terrain, le chien et la femme communiquent aisément. Canine doit récupérer un lot de passeports français vierges volés par des mafieux corses, mais également convoités par les Russes et les Américains - la Guerre froide n'est pas loin. Gunn, très conscient de ses qualités, n'accepte pas d'être traité comme un chien ordinaire :

Oh le bon gros toutou qui suit la piste aux vilains messieurs ! (...)

Les oreilles de son compagnon se couchèrent sur sa nuque; il fit semblant de ne pas entendre et se concentra sur la piste à suivre. Il ne pouvait pas supporter ce langage enfantin qu'emploient habituellement les gens avec leurs animaux de compagnie ; il jugeait cela indigne de sa maîtresse et de lui-même. (Oppel-Dorison, $1983: 27$ )

Gunn sait aussi barrer un voilier. Canine lui impose un quart de nuit : « il veillerait les bateaux pour prévoir un risque d'abordage, humerait les variations du vent pour prévoir l'arrivée d'un grain et surveillerait le cap. » Il sait même lire la date de son anniversaire et le rappelle à Canine qui a oublié. Ce chien mime l'homme et le remplace dans les limites du vraisemblable si l'on considère qu'un dressage poussé puisse permettre de tels résultats.

12 La figure qui prime dans ce roman des années 1980 est celle du chien offensif, adaptable, chasseur, combattant d'une cause juste, allié fidèle de l'homme, ici d'une femme, choix qui n'est pas anodin, et enfin symbole de puissance sexuelle.

13 La queue en trompette, San Antonio, de Frédéric Dard, publié en 1997. San Antonio est un personnage majeur du polar français. Il a été créé en 1949 par Frédéric Dard (175 titres jusqu'en 2001), collection reprise à sa mort, en 2000, par son fils, Patrice Dard. Commissaire de police à Paris, San Antonio est secondé par les inspecteurs Bérurier et Pinaud. Nous retrouvons un langage populaire, des néologismes, des jeux de mot, de l'humour grivois cette fois, et des distorsions de citations littéraires. Les animaux sont peu présents chez Dard et La queue en trompette est une originalité qui n'a pas été renouvelée parce qu'elle n'a pas été du goût d'un public d'habitués. En effet, les comportements de Salami en font un chien très anthropomorphisé. Il est un " personnage » du roman car s'il est le compagnon du commissaire, ce basset hound est aussi un enquêteur à part entière. Sans lui, l'enquête n'aboutissait pas. De plus, Salami sauvera San Antonio au sens propre comme du ridicule.Achille, commissaire à la retraite, qui a confié le chien à San Antonio en dit :

(...) Salami est in-tel-li-gent. Vraiment intelligent! Comme vous l'êtes, comme je le suis moi-même. Il comprend ce qu'on lui dit. Il suffit de lui consacrer quelque attention pour s'apercevoir qu'il pense. Vous avez déjà rencontré un chien qui pense ? Pour ma part, c'est le premier. (Dard, $1997: 17$ )

14 Au cours de leur première rencontre, c'est plutôt le chien qui sonde l'homme que le contraire. Et Salami exige d'être vouvoyé. San Antonio confie que « en lui accordant cette promotion, je le faisais accéder au statut unique de "chien humain ». Comme dans Canine et Gunn, l'homme et l'animal dialoguent mais après avoir mis au point ici une méthode plus sophistiquée : San Antonio émet un avis, ou fait une proposition et le chien répond d'un geste par oui ou par non. C'est un peu long mais cela fonctionne. 
Quand il est en désaccord avec son maître, Salami adopte son "attitude Rodin ", celle du célèbre Penseur. Lors de la phase de résolution finale de l'enquête, Salami s'installe :

Un qui me passionne, c'est le signore Salami !

$\mathrm{Tu}$ le verrais suivre l'interview, tu le prendrais pour un juge britannique emperruqué et tu l'appellerais "Votre Honneur ». Il possède une chose commune aux Lords : il adore les sièges. La façon dont il pratique un fauteuil est révélatrice de son sens de l'humain. (Dard, $1997: 275$ )

Salami est donc hissé au niveau de l'humain, imitant, singeant les comportements des hommes entre eux. Il est un miroir des humains. Quand il résout l'énigme, San Antonio en partage le mérite avec son "adjoint quadrupède ». Dès que San Antonio et lui ont trouvé un moyen de communiquer, il devient un véritable partenaire du commissaire. Et Salami a le même foutu caractère que les humains, avec des caprices (le vouvoiement), un grand appétit pour le sexe. Il est un San Antonio fait chien pour mieux le moquer. C'est le héros animalier qui surpasse l'homme dans la déduction, le renvoyant à la seule action dans cette enquête emberlificotée. La fonction du chien est aussi celle $\mathrm{du}$ fou de la cour, qui amuse mais qui assène quelques vérités.C'est également le rôle de ce roman parodique que de malmener non pas le système - ce n'est pas un roman noir - mais les relations interpersonnelles entre représentants du genre humain.

Benoit Séverac, Georges Simenon et Frédéric Jaccaud présentent, eux, des chiens perdus, victimes et bourreaux des hommes, porteurs de chaos annoncés, individuels ou collectifs. Le chien arabe de Benoit Séverac est publié en 2017. Un titre qui a posé problème à l'éditeur car l'association chien et arabe est blasphématoire pour la communauté musulmane. Le chien est un animal impur comme le porc. D'autant plus que Benoit Séverac nous entraine dans un quartier pluricommunautaire sensible de Toulouse. C'est un roman reportage à la manière du roman noir brésilien (José Louzeiro, Paulo Lins, Edyr Augusto), une plongée dans une cité hostile et dangereuse où règnent les dealers de drogue.

Samia implore Sergine, vétérinaire dans le quartier voisin, de sauver un chien malade qui appartient à son frère. Elle est la petite sœur d'un caïd qui utilise des rottweilers comme passeurs de cocaïne depuis l'Espagne. Ce nouveau trafic qui avilit une jeunesse qu'ils préféreraient orienter vers le Jihad ne plait pas non plus aux intégristes musulmans. La jeune vétérinaire va se lancer dans une croisade imprudente pour sauver ces animaux victimes des trafiquants, et ses initiatives vont précipiter un embrasement général du quartier.

18 Le rottweiler, animal dangereux dont la possession est très réglementée, est ici réduit au statut de victime, de moyen de transport d'une substance illicite, d'objet. Il est dressé, soumis, torturé. Quand Samia, 16 ans, va confier à Sergine qu'elle est promise en mariage en Algérie, la vétérinaire va comparer son sort à celui du chien :

Quoi, le chien?

Tu as bien désobéi à ton frère en me le confiant. Comme quoi les lois que ta famille t'impose, tu sais les contourner parfois.

C'est pas pareil. Il est innocent, lui.

Pourquoi? Tu as fait quelque chose de mal, toi?

Un chien ne peut pas se défendre.

Toi non plus visiblement. Si je peux aider un chien, je peux t'aider, toi. (Séverac, 2017 : 36) 

curieux chien jaune s'invite à plusieurs moments de l'intrigue, semant le trouble parmi la population. A qui appartient-il ? Pourquoi est-il là ? Sa présence pourra-t-elle aider à expliquer des crimes qui se succèdent? Le chien est présent à côté de la première victime : «Le douanier s'avance vers l'homme couché, un peu rassuré par la présence du chien, une grosse bête jaune et hargneuse. " (Simenon, 1936:10) L'homme court prévenir à l'hôtel de l'Amiral. «Le douanier se retourne. Le chien jaune est entré sur ses talons et s'est couché aux pieds de la fille de salle. » (Simenon, 1936 : 11) Il est clair que la présence de ce chien auprès de la victime et de la serveuse du café est un indice proposé au lecteur et au commissaire Maigret :

Le regard de Maigret tomba sur un chien jaune, couché au pied de la caisse. Il leva les yeux, aperçut la jupe noire, un tablier blanc, un visage sans grâce et pourtant si attachant que pendant la conversation qui suivit il ne cessa de l'observer. (Simenon, $1936: 17)$ intrigue Maigret qui va interroger le journaliste de Concarneau :

Personne ne sait d'où il sort... On a cru un moment qu'il appartenait au caboteur arrivé hier... Le Sainte Marie... Il parait que non... Il y a bien un chien à bord mais c'est une terre-neuve, tandis que je défie qui que ce soit de dire de quelle race est cette affreuse bête... (Simenon, $1936: 19$ ) 
autre crime est commis. Le chien jaune devient le symbole de la peur qui s'étend sur la ville. Il représente l'étranger qui doit être chassé de la communauté à coup de fusil. Un cordonnier lui tire dessus, les habitants lui jettent des pierres. Ils auraient fait subir le même sort à un homme qui n'est pas d'ici, un vagabond. Au chien jaune est associée la présence d'un mystérieux rodeur. Le docteur Michoux, le denier survivant du groupe des notables s'en ouvre à Maigret :

(...) Je m'attendrais à ce que ce soit lui qui vienne, l'homme au chien, le fou, l'assassin.

Un peu plus et il claquait des dents. Il ajoute :

Pensez-vous que vous allez l'arrêter ou l'abattre comme un animal enragé ?... Car il

est enragé !... On ne tue pas comme ça sans raison... » (Simenon, $1936: 98$ )

decteur Michoux fond dans le même moule le chien et l'homme, le chien dit « enragé » qui a communiqué sa « rage » à l'homme. Tuons l'homme ! Même après avoir disparu, le chien jaune catalyse les peurs. Michoux avec ses amis les notables avaient escroqué Léon, le rodeur de retour, l'envoyant en prison pour vingt ans. Léon était patron pêcheur et le chien jaune était son chien de bateau. De retour à Concarneau, le chien est son messager : je reviens, signifie-t-il aux escrocs.

Le chien jaune a une figure double. Il est pour son maître le compagnon fidèle, le garant d'une moralité qui retient la vengeance. Il est aussi le symbole de la justice, de la patience dans l'attente d'un retour à l'ordre, et du châtiment de ceux qui ont commis un crime contre son maitre. Pour la population de la ville, il est l'annonciateur d'une perturbation sociale: attention, les cadavres vont sortir des placards, les coupables d'antan vont trembler et devoir régler des comptes. Le chien est le signe d'une menace collective diffuse, puis de plus en plus précise. La couleur jaune est traditionnellement celle de la fourberie, de la traîtrise. A contrario, le retour de ce chien jaune-là est celui du retour du bon droit. Il vient se coucher au pied d'Emma qu'il désigne au commissaire comme la personne qui est au centre de l'énigme. Sa seule présence et la crainte qu'il inspire aux escrocs d'hier va déclencher une réaction en chaine d'auto-élimination du groupe qui était à l'origine de l'emprisonnement de son maître.

Hécate, faits divers, de Frédéric Jaccaud a été publié en 2014. L'auteur est suisse, de Lausanne, critique littéraire et romancier. Hécate, fait divers est son troisième roman. Le titre étonne et annonce un texte à double niveau par la juxtaposition du nom d'une divinité maléfique entourée de chiens monstrueux et de l'expression "fait divers ", quelque chose de peu d'importance. Effectivement, Hécate est un roman très sombre, très violent, et très référencé culturellement.

29 Anton, un jeune flic, de ceux des policiers qui règlent la circulation, s'introduit sur une scène de crime épouvantable: le corps nu d'un médecin, Sacha, déchiqueté par ses chiens dans une villa cossue de Ljubljana. Voici comment sont décrits les trois bullmastiffs à leur sortie de la maison :

A travers les muselières, on aperçoit les gueules massives et humides des canidés; d'épais fils blanchâtres coulent jusqu'au sol. Les babines pendantes, qui leur donnent cet air presque triste, se révèlent et exhibent des dents effrayantes, des canines jaunies, jusqu'à leur gencive rouge. (...) Ils n'ont pas l'élégance des félins, cette ondulation feutrée, mais au contraire des mouvements qui expriment la force brute, la bestialité ; il s'agit de quelque chose d'ancestral qui végète au plus profond du compagnon idéal depuis toujours. (Jaccaud, 2014 : 13-14)

Ces trois chiens renvoient à Cerbère, le chien tricéphale gardien des Enfers chargé d'empêcher les morts de s'échapper et d'interdire aux vivants de venir y enlever des 
morts qui leur sont chers. Ils font aussi référence aux trois têtes de la déesse Hécate correspondant chacune à une attribution : reine de la nature, divinité protectrice des enfants et déesse de la lune. C'est cette attribution nocturne qui, avec le temps, va l'emporter sur les deux autres : déesse de la magie et de la divination, elle s'entoure de chiens monstrueux chargés de terroriser l'âme des meurtriers.

Anton est fasciné par le « cadavre mutilé et impudique » de Sacha :

Il ne s'agit pas ici d'un homme assassiné, d'un homme torturé, mais d'un corps meurtri par des dents et des griffes, un cadavre primal, premier, non pas mangé mais déchiqueté, tel qu'on pouvait en rencontrer dans les forêts obscures de cette humanité d'avant, de cette humanité qui tremblait en entendant hurler les loups, grogner les meutes affamées de chiens, effrayée de pouvoir être pourchassée. (Jaccaud, $2014: 26$ )

Anton va vite comprendre que ce carnage a été mis en scène par la victime, qu'il s'agit d'un rite zoophile qui a mal tourné. Au mur de la pièce, il y a une reproduction d'un tableau de William Blake, Hécate ou la nuit de joie d'Enitharmon. Troublé par le cadavre comme par le tableau, Anton va vouloir comprendre ce qui s'est passé. Son esprit peu préparé va vite être dépassé par sa quête, car il est un jeune un peu naïf et sans culture séduit par une démarche sectaire. Il va se laisser enfermer dans une logique criminelle.

Alors Anton reconstitue l'histoire de Sacha dans son petit carnet de notes qu'il commence par: " 1974. Ici se cache l'origine de l'obscène ». Une petite fille, Milena, 7 ans. Un père, trois frères qui la caressent déjà. La route nationale, les camions. Pour distraire les routiers, les grands frères organisent de combats de chien et prostituent leur petite sœur. Un jour, Milena refuse de coucher avec les clients si ses frères font combattre les chiens. Ils la suspendent au-dessus de l'enclos des molosses et elle se fait mordre. Elle aimerait être un de ces chiens et dévorer ses frères, sa famille, tout le village. Puis Milena est « vendue » à un alsacien d'âge mûr, tombe enceinte, tue l'enfant qu'elle porte, divorce, se fait payer des études de médecine par son ex-mari et décide de changer de sexe. Milena est devenue Sacha, médecin à Ljubljana.

Hécate devenue mâle tourne le dos à sa meute. De retour au pays natal, dans cette utopie de boue, Sacha veut prendre sa revanche. Il s'est couvert de poils, s'est transformé en démon, en chien et veut hurler à la lune. Mais il découvre un pays qui ressemble à tous les autres. Les villages n'y sont pas infernaux, toutes les petites filles ne sont pas violées; il n'y a aucun combat de chiens. (...) Sous son masque viril, il est convaincu que les chiens ne le retrouveront pas.

Une nuit pourtant, les aboiements recommencent à résonner dans ses rêves. (Jaccaud, 2014 : 99)

34 Maintenant Anton étudie le tableau de William Blake qui est selon lui la clé pour comprendre le passage à l'acte de Sacha. Lubja, sa compagne, est sidérée par l'évolution d'Anton qui lui explique :

Qui sont les chiens? Le regard, les autres, les proclamateurs de différence, mais aussi celui qui essaie d'échapper à son destin, qui cherche à comprendre, qui cherche dans les textes, dans les peintures. Nous sommes tous des chiens, dit Anton. (...) En acceptant d'endosser le rôle d'Hécate, Sacha a tué tous les hommes qui l'ont sali. Milena s'est rendu justice à elle-même en se laissant dévorer. (Jaccaud, $2014: 114$ )

Anton veut reproduire l'acte de Sacha. Il attire Lubja dans sa chambre ornée de la reproduction du tableau. Trois jours plus tard, la police libèrera Lubja des tortures infligées par Anton. Au tribunal, l'avocat de Lubja récuse la folie. Pour lui, Anton s'est laissé fasciner par la scène du suicide de Sacha et a tenté d'y apporter du sens, mais en 
s'y vautrant. Pour les psychiatres, Anton est un esprit faible mis face à une situation complexe qui a dépassé ses capacités d'interprétation. "Il est allé au-delà du fait divers ».

La figure du chien dans Hécate, fait divers, est complexe et particulièrement maléfique. Il y a d'abord les chiens victimes, au village, dressés pour combattre comme Milena est dressée pour la prostitution. Et Milena rêve alors de devenir chien et mâle pour inverser les rôles et le pouvoir. La dimension sexuelle de la représentation du chien est permanente. Puis il y a les trois chiens de Sacha-Milena, victimes eux aussi des sévices que leur maître leur inflige enfin, à son tour, se vengeant du passé. Les chiens de Sacha vont se révolter et déchiqueter Sacha. Ils renvoient également au symbole du psychopompe, le guide du passage des âmes vers le royaume des morts. Ils sont des passeurs $\neg$ brutaux, certes $\neg$ mais des passeurs malgré tout entre le monde des vivants et l'au-delà.

米

En conclusion, nous examinerons comment les fonctions et les figures du chien de notre corpus communiquent avec les sous-genres du roman policier. Reprenant ce qu'avait ébauché Tzvetan Todorov dans Poétique de la prose en 1971, Yves Reuter organise la littérature policière en trois sous-genres : le roman à énigme, le roman noir et le roman à suspense que Boileau-Narcejac définira comme le roman de la victime.

Le roman à énigme est le roman de l'enquête. Il propose une structure bien définie : crime, investigation, résolution. Le détective et le criminel s'affrontent par un jeu de déductions et de fausses pistes; le roman noir ou le roman de l'enquêteur qui est aux prises avec un univers hostile le plus souvent urbain et avec ses démons personnels (failles passées, solitude, alcool...). L'intérêt est plus dans l'atmosphère et dans la critique de la société que dans l'intrigue elle-même ; le roman d'angoisse et de suspense qui est le roman de la victime, celui d'un individu ordinaire confronté à une situation extraordinaire, inattendue et très angoissante.

Deux romans sont des romans à énigme: La queue en trompette dans une veine parodique et Le chien jaune avec une orientation plus noire. Salami et le chien jaune de Léon, le marin pêcheur, sont les compagnons fidèles de leurs maîtres. Le premier est un acteur de l'enquête du commissaire. Le second est un messager de justice, annonçant le retour de Léon, celui qui a été floué vingt ans auparavant par un petit groupe de notables de Concarneau. Les deux romans qui présentent un chien dont la seule fonction est l'accompagnement de leur maître, La nuit myope et Tequila frappée, sont des romans noirs, un noir tendre, le premier tend vers l'angoisse, le second a un ton clairement parodique. Les maîtres de ces chiens sont des hommes désorientés, des losers, personnages caractéristiques du noir: Domi, le bourgeois myope qui traverse Paris la nuit sans ses lunettes pour rejoindre celle avec qui il veut changer de vie, et l'inspecteur Lynch, divorcé, alcoolique, solitaire et subissant ses enquêtes plus qu'il ne les conduit. Dans Canine et Gunn, l'animal est un clone de son maître agent secret. Les figures de chien compagnon dans La nuit myope et Tequila frappée se calquent sur les topos de leurs maîtres, des personnages du roman noir souvent inaptes au bonheur. Nous sommes à la lisière est une phrase de Pierre Gascar que Caroline Lamarche a choisie comme titre de son recueil de nouvelles. La narratrice s'adresse à son animal préféré, une cane : «Le moment est venu du choix entre les humains et ta vraie vie, celle pour 
laquelle tu es née» (Lamarche, 2019: 57). Cane ou cheval, ou chien, la relation entretenue rapproche les deux mondes animal et humain. Les quatre chiens de ces quatre romans refusent de choisir. Le sens de leur présence est la complémentarité de leur fonction avec celle de l'homme: fou du roi, le chien singe l'homme pour le renvoyer à plus d'humilité ; compagnon fidèle, il est un garde-fou contre ses faiblesses, il adoucit ses ruptures passées.

Le roman noir à très noir réunit Le chien arabe et Hécate, fait divers, dont les chiens sont à la fois victimes et porteurs de mort, voire bourreaux comme dans Hécate. Ces chiens maléfiques sont associés à la mort, parce qu'ils l'annoncent ou la donnent, parce qu'ils ont été dressés pour cela par les hommes; ils rejoignent les romans noirs et leurs univers sombres et violents, qu'ils soient urbains ou ruraux.

Tableau des correspondances Fonction - Figures du chien - Sous-genres du roman policier

\begin{tabular}{|c|c|c|c|c|c|}
\hline \multirow[t]{2}{*}{ titre } & \multirow[t]{2}{*}{ fonction } & \multirow{2}{*}{$\begin{array}{l}\text { figures } \\
1-2-3\end{array}$} & \multicolumn{3}{|c|}{ sous-genre } \\
\hline & & & énigme & noir & angoisse \\
\hline La nuit myope & $\begin{array}{l}\text { compagnon du } \\
\text { personnage }\end{array}$ & $\begin{array}{l}1 \\
\text { Gardien confident conseiller }\end{array}$ & & $1 /$ noir & 2/angoisse \\
\hline Tequila frappée & $\begin{array}{l}\text { compagnon du } \\
\text { personnage }\end{array}$ & 1 Gardien confident conseiller & & $\begin{array}{l}\text { Noir } \\
\text { parodique }\end{array}$ & \\
\hline Canine et Gunn & $\begin{array}{l}\text { compagnon et } \\
\text { enquêteur }\end{array}$ & $\begin{array}{l}1+2 \\
\text { Gardien confident conseiller } \\
\text { Chasseur courageux }\end{array}$ & & $\begin{array}{l}\text { noir daté } \\
\text { parodique }\end{array}$ & \\
\hline $\begin{array}{l}\text { La queue en } \\
\text { trompette }\end{array}$ & $\begin{array}{l}\text { compagnon et } \\
\text { enquêteur }\end{array}$ & $\begin{array}{l}1+2 \\
\text { Gardien confident conseiller } \\
\text { Chasseur courageux }\end{array}$ & $\begin{array}{l}\text { énigme } \\
\text { parodique }\end{array}$ & & \\
\hline Le chien arabe & $\begin{array}{l}\text { acteur } \\
\text { indépendant } \\
\text { maléfique }\end{array}$ & $\begin{array}{l}3 \\
\text { Victime } \\
\text { porteur de mort } \\
\text { annonceur de perturbation sociale }\end{array}$ & & $\begin{array}{l}\text { noir } \\
\text { reportage }\end{array}$ & \\
\hline Le chien jaune & $\begin{array}{l}\text { compagnon et } \\
\text { acteur } \\
\text { indépendant } \\
\text { positif }\end{array}$ & $\begin{array}{l}1+3 \\
\text { Gardien fidélité } \\
\text { annonceur de perturbation sociale }\end{array}$ & 1/énigme & & $2 /$ noir \\
\hline Hécate, fait dlvers & $\begin{array}{l}\text { acteur } \\
\text { indépendant } \\
\text { maléfique }\end{array}$ & $\begin{array}{l}3+ \\
\text { Victime } \\
\text { Monstre férocité } \\
\text { annonce et donne la mort }\end{array}$ & & $1 /$ noir ++ & 2/angoisse \\
\hline
\end{tabular}

41 Aucun de ces sept romans cynophiles ne présente une majeure d'angoisse et de suspense. Mais deux d'entre eux montrent une dimension angoissante importante car ils mettent en danger un personnage confronté à une situation particulièrement déstabilisante : Domi perdu dans Paris en compagnie de son chien et Anton, le jeune policier qui s'enferme dans un processus psychologique le conduisant au crime. Hécate est à ce titre un roman de la victime.

Le roman policier pour la jeunesse, un sous-genre en plein essor aurait mérité d'être également étudié. Le chien y est fortement représenté soit comme victime défendue par les enfants, soit surtout comme gardien, protecteur et acteur des enquêtes qu'ils mènent. Dans Pire que terrible d'Alain Gagnol et Jean-Loup Felicioli, le père d'un des jeunes héros dit: «Les chiens sont comme nos miroirs. Ils s'adaptent tellement aux hommes qu'ils finissent par leur ressembler comme leur ombre. » Ce n'est pas toujours à notre avantage. 


\section{BIBLIOGRAPHIE}

A.D.G (1990). La nuit myope. Paris : Balland.

BOILEAU-NARCEJAC (1975), Le roman policier. Paris : Presses Universitaires de France.

DARD, Frédéric (1997). San-Antonio, La queue en trompette. Paris : Fleuve Noir.

GAGNOL, Alain et FELICIOLI Jean-Loup (2005). Pire que terrible. Paris : Magnard Jeunesse, Policier.

GASCAR, Pierre (1953). Les bêtes. Paris : Gallimard.

JACCAUD, Frédéric (2014). Hécate, fait divers. Paris : Gallimard, Série Noire.

LAMARCHE, Caroline (2019). Nous sommes à la lisière. Paris : Gallimard.

MESPLEDE, Claude (2007). Dictionnaire des littératures policières. Nantes : Joseph K.

MONFILS, Nadine (2016). Tequila frappée. Paris : Belfond.

opPEL et Dorison (1983). Canine et Gunn. Paris : Gallimard, Série Noire.

REUTER, Yves (2012). Le roman policier. Paris: Armand Colin.

SÉVERAC, Benoit (2017). Le chien arabe. Paris : La manufacture de livres.

SIMENON, Georges (1936). Le chien jaune. Paris : Fayard, Le Livre de Poche.

TODOROv, Tzvetan, (1971). « Typologie du roman policier » in Poétique de la prose. Paris, Seuil, pp. 55-64.

\section{RÉSUMÉS}

Dans le roman policier aujourd'hui, le chien n'est plus l'animal obéissant, l'assistant muet de son maître qui utilise son odorat comme le détective se sert de sa loupe. Le chien du roman policier contemporain n'est plus le chien policier des films pour la jeunesse des années 1950 : il réfléchit, il communique avec son maître, il le conseille, il le console. Il s'éloigne du genre animal et se rapproche de l'homme.Nous observerons cette figure du chien bienfaisant plutôt dans des romans à énigme où l'intrigue est construite et où le personnage de l'enquêteur tient le devant de la scène. Dans le roman noir à l'univers hostile, le chien n'est plus l'allié de l'homme. Il est sa victime ou son bourreau; il annonce un danger, un bouleversement ou la mort. C'est un animal féroce, maléfique. Finalement, dans un cas comme dans l'autre, il n'est que la voix de son maître, le miroir de l'homme et celui de la société.

In today's detective story, the dog is no longer the obedient animal, the mute assistant of its master which uses its smell as he uses his detective magnifier. The dog of the contemporary detective novel is no longer the police dog we saw in the films for young people in the fifties: it thinks, it communicates with its master, gives advice to him and comforts him. It is more and more distant from the animal and closer to humans. We will find this figure of the salutary dog more in where the plot is built and the character of the detective is at the front of the stage. In the noir novel with its somber atmosphere, the dog is no longer men's friend. It is its victim or its executioner. It announces danger, disruption or death. It is a fierce, evil animal. Eventually, in all cases, it is nothing but its master's voice, the mirror of mankind and society. 
INDEX

Mots-clés : roman policier, chien, enquêteur, compagnon, victime, bourreau

Keywords : detective story, dog, detective, companion, victim, executioner

\section{AUTEUR}

\section{PIERRE MICHEL PRANVILLE}

CREPAL Sorbonne Nouvelle Paris 3

piermichelpranville[at]free.fr 